\title{
Application of Gamma Process for Deterioration Prediction of Buildings from Discrete Condition Data
}

\author{
Ruwini Edirisinghe, Sujeeva Setunge and Guomin Zhang \\ RMIT University, Melbourne, Australia
}

\begin{abstract}
Deterioration prediction of civil infrastructure from discrete condition data is a challenge faced by many asset managers developing effective maintenance and renewal strategies. Due to high variability of data, often, deterministic methods are not readily applicable. Some of the reliability based methods adopted are time dependent reliability analysis, such as Markov chain and more recently gamma process. Whilst such models have been developed for assets with smaller number components, such as bridges and storm water pipes, for complex systems such as buildings reliability based methods are less common. The second largest class of infrastructure assets the local governments own in Australia is the community buildings. As the majority of existing community buildings are maturing, the local governments seek more reliable asset management strategies. Condition based forecasting is a major component of such asset management approaches. This paper presents development of a reliability based methodology for deterioration prediction of community buildings. Gamma process is considered to be an appropriate approach for predicting building element deterioration due to the temporal variability of degradation. The Gamma deterioration process presented in this paper is a stochastic process with independent non-negative increments having gamma distribution with identical scale parameter. Building inspection data from one of the local governments in Victoria are used in the model. Further, analysis of the data and model results are discussed.
\end{abstract}

Key words: Deterioration prediction, Gamma process, building management.

\section{Introduction}

Infrastructure assets belonging to Victorian Local Governments (LGs) represent a vast investment built up over many generations, and are valued at approximately 23.3 billion dollars [1]. Out of these, community buildings are the second largest class of assets. Building management is challenging due to the high level of 
complexity of the buildings unlike the other asset classes such as roads. Above and beyond, the number of aging community building infrastructure the LGs own is growing exponentially. Consequently, the municipalities are in need of reliable building management strategies.

A research project funded by the Australian Research Council (ARC) entitled "A reliability based approach for sustainable management of community buildings" has six Victorian municipalities as partners. One of the requirements of the partner councils is to develop a sophisticated and more reliable deterioration prediction mechanism in contrast to the default linear deterioration curves they currently use for building assets management. Further, currently this default deterioration curve is not analyzed against each building element or the whole building rather is used as a snapshot for the whole set of buildings the council owns. Moreover, the current decision making strategies for buildings most of these councils practice are reactive or on an as-needed basis rather than a proactive strategy with the understanding of the future needs. Hence, a more reliable deterioration prediction method for individual building elements is an industry need and a gap to be filled.

Deterioration prediction is a significant stage in whole of life building management process [2]. In brief, the whole building management process is divided in to six stages as Infrastructure System and Elements Hierarchy, Condition Rating Method, Data Collection Method, Deterioration Prediction Method, Cost Forecasting and Decision Making. The STAGE 4 of building management process is Deterioration Prediction Method and it focuses on the deterioration prediction of the individual elements of the building and/or the building as a whole infrastructure system based on the condition data from STAGE 3.

The focus of this paper is to derive and analyze a reliability based deterioration prediction model based on the building condition inspection data from one partner council of the research project.

The rest of the paper is organized as follows. Section 2 presents the literature review of the deterioration prediction mechanisms used for various infrastructure asset management approaches. Section 3 discusses the gamma process model which is a stochastic deterioration prediction method that considers the temporal variability nature of the deterioration rate of infrastructure. Section 4 presents the analysis of the gamma process deterioration prediction model based on the building inspection data collected by the Victorian council. The data preparation, model parameter 
estimation, probability distribution and deterioration prediction are discussed. Finally, Section 5 concludes the paper.

\section{Related Work}

Various infrastructure deterioration prediction models have been discussed in the literature. The existing deterioration models for infrastructure assets can be broadly classified into three categories as deterministic models, statistical models and Artificial Intelligence (AI) based models.

Deterministic deterioration prediction models can be of two types: linear or nonlinear. Deterministic models are often used for phenomenon where relationships between components are certain. Time linear and power law models are applied for water mains [3] and pavements [4]. Exponential deterministic models are applied for pipes [5-7]. The deterministic models, if can be fitted accurately can be analyzed in mathematical formulae and the relationship between input and output parameters is straight forward. However, this approach is too simplistic to reflect the probabilistic nature of the deterioration particularly with the data with random noise.

Among the AI based techniques, Case-Based Reasoning (CBR), fuzzy set theory and Neural Networks (NNs) are used for modeling the deterioration of infrastructure facilities. CBR is used for sewer [8]. Fuzzy set theory is used in buried pipes [9]. NNs are used to model deterioration of various infrastructure assets including sewers [10, 11 ], bridges [12], oil and gas pipelines [13], concrete structures [14] and storm water pipes [15]. Some of the AI based models are of the data-driven type in which model structure is determined by data, i.e. no assumptions are made regarding the model structure [15]. Even though, AI based approaches are insensitive to noisy data and can automatically detect non-linear underlying models, they have a demand for large quantities of data and are less likely to have an underlying model that describes the process hence, leading to a danger of over fitting [15]. Statistical modeling is based on statistical theory for modeling phenomenon where random noise in components exists and uncertainty is in-built. Likelihood that condition of an infrastructure facility will change from one state to another is probabilistic in nature because the infrastructure deterioration cannot be predicted with certainty due to unobserved explanatory variables, the presence of measurement errors and the inherent stochasticity of the deterioration process [16]. Hence, it is more appropriate to base the deterioration modeling of engineering structures and infrastructure in terms of a time-dependent stochastic process. 
In these time-dependent models the co-efficients such as average rate of deterioration per unit time are random variables [17]. However, these randomvariable models do not take the temporal variability associated with the deterioration rate in to account. Hence, the need for Markov process based models for deterioration modeling has been noted in the literature [18].

Discrete time Markov process and continuous time Markov process are two variants of Markov process used in statistical deterioration prediction modeling. Markov chain is a discrete time Markov process and is used to predict deterioration in bridges [19], sewers [20] and storm water pipes [15, 21] in the literature. Markov model based decision making process together with the building weighting is suggested [22]. Compound poisson process and gamma process are variants of continuous time Markov process that can be used in deterioration modeling. According to Singpurwalla and Wilson, the main difference between these two is that compound poisson process has a finite number of jumps in finite time intervals, whereas gamma process has an infinite number of jumps in finite time intervals [23]. The compound poisson processes is suitable for modeling usage such as damage due to sporadic shocks [17]. The gamma process in contrast, is more suitable for modeling gradual damage accumulating over time in a sequence of increments by continuous use.

As deterioration is generally uncertain and non-decreasing, it can best be regarded as a gamma process [24] which gives a proper model for random deterioration with time. Gamma process is used to model the uncertainty in the time to failure (lifetime) and/or the rate of deterioration. Gamma processes are fitted to data on creep of concrete [25], fatigue crack growth [26], thinning due to corrosion [27] and corroded steel gates [28]. Further, gamma process has been used in bridge deterioration modeling [29, 30].

Even though Markov chain has been widely used to model the deterioration of various infrastructure assets, the process has been criticized and the restrictive stationary assumptions about the time dependent deterioration rate in Markov chain are being argued in the literature [16, 31]. There are reasons for these arguments. Firstly, in Markov chain the deterioration is assumed to be a single step function. That is the elements do not transit more than one condition state with in a given time step period. Secondly, the transition probabilities in Markov chain are not time 
variant and cannot capture the temporal variability associated with the evolution of the deterioration. Thirdly, the unit of time or the inspection period which is the time between two consecutive inspections is same in the Markov chain method.

\section{Gamma Process Deterioration Model}

As discussed above, the gamma process is the most appropriate mechanism to model the monotonic and gradual deterioration occurring in building components. The gamma process is a stochastic process with independent, non-negative increments having a gamma distribution with an identical scale parameter.

\subsection{Non stationary Gamma Process}

In the gamma process deterioration model, the cumulative deterioration $\mathrm{X}$ is a random quantity. At time $t$ it has a gamma distribution with the shape function $a(t)>0$ and the scale parameter $b>0$ which is a constant. The Probability Density Function (PDF) of $\mathrm{X}$ is given by

$$
G a(x \mid a(t), b)=f_{X(t)}(x)=\frac{b^{a(t)}}{\Gamma(a(t))} x^{a(t)-1} e^{-b x} I_{(0, \infty)}(x)
$$

Where, $I_{A}(x)=1$ for $x \in A$ and $I_{A}(x)=0$ for $x \notin A$ and $\Gamma(u)$ is the gamma function for $u>0$ which is given by

$$
\Gamma(u)=\int_{v=0}^{\infty} v^{u-1} e^{-u} d u
$$

The function $a(t)$ must be an increasing, right-continuous real-valued function of time $t$, with $a(0) \equiv 0$ to facilitate the monotonic nature of deterioration over time.

$\{X(t), t>0\}$, the continuous-time stochastic gamma process has independent increments. i.e. Let the cumulative deterioration at time $t_{1}$ and $t_{2}$ are $X\left(t_{1}\right)$ and $X\left(t_{2}\right)$, respectively.

The deterioration increment from time $t_{1}$ to $t_{2}$ be independent of the cumulative deterioration at time $t_{1}$ and it is a non-negative quantity. i.e. $X\left(t_{2}\right)-X\left(t_{1}\right)$ is independent of $X\left(t_{1}\right)$. Further,

$$
X\left(t_{2}\right)-X\left(t_{1}\right) \sim G a\left(a\left(t_{2}\right)-a\left(t_{1}\right), b\right)
$$

The gamma Cumulative Distribution Function (CDF) of the damage is denoted as

$$
G A(y \mid a(t), b)=\int_{0}^{y} G a(x \mid a(t), b) d x
$$


The mean, variance and coefficient of variation of the cumulative deterioration at

$$
\begin{gathered}
\text { time } t \text { are given as } \quad \mu_{X(t)}=\frac{a(t)}{b}, \quad \sigma_{X(t)}=\frac{\sqrt{a(t)}}{b}, \quad \text { and } \\
v_{X(t)}=\frac{1}{\sqrt{a(t)}}
\end{gathered}
$$

Where coefficient of variation is a time-dependent function and it is inversely proportional to the time.

\section{Data Analysis}

\subsection{Condition Data}

The council building condition data of inspections conducted in years 2007 and 2009 are used in our model. The element hierarchy used composed of two levels. The first level building elements are Essential Services, Finishes, Fittings, Risks, Services, Substructure and Superstructure. The condition rating used in building inspection is 1-10 rating where 1 corresponds to near new asset and 10 corresponds to an asset that has failed and is no longer serviceable. The condition audits were conducted in 2007 on 139 buildings and in 2009 on 209 buildings. Out of which there are 77 buildings of which inspection data are common to both years and be used in the model.

The elements are categorised in to three groups according to the inspection data. Let the element condition in most recent inspection be $C_{c}$ (current condition) and the condition in previous inspection be $C_{p}$ (previous condition). Then an element can be in one of the following three states $S_{1}, S_{2}$ or $S_{3}$ which are mutually exclusive and exhaustive states defined based on two consecutive element conditions. The states are $S_{1}$, where $C_{c} \geq C_{p} ; S_{2}$, where $C_{c}<C_{p}$ and $S_{3}$, where $C_{c}=C_{p} . S_{2}$ represents a state where the element has been renewed or refurbished as the current condition is improved compared to the previous condition. $S_{3}$ represents a state where the element has not been deteriorated as the condition remains the same. $S_{1}$ represents a state where the element has been actually deteriorated as the current condition is poor compared to the previous condition. The gamma process is used to model the incremental deterioration over time. Hence, the non-incremental datasets 
corresponds to states $S_{2}$ and $S_{3}$ are omitted in this study. The dataset corresponds to the state $S_{1}$ that exhibits the positive increments are used in our gamma process model.

There are 339 building element data points common to two years, 2007 and 2009 that corresponds to the criteria of condition state $S_{1}$. The building element Superstructure is used in the gamma process model that has 151 common data points.

\subsection{Deterioration Data}

As the gamma process model is used for the damage or the deterioration, the inspection data are to be adjusted to derive the deterioration of the building element under consideration. Hence, the condition data are adjusted so that,

AccumulatedDeterioration $=$ condition -1

For example, a brand new element which is in condition 1 has the accumulated deterioration 0 and a failed element which is in condition 10 has the accumulated deterioration 9. Hence, from this point onwards the paper refers to the deterioration rather than the condition of the element.

\subsubsection{Deterministic Approach}

In 2002 a financial valuation and building audit was carried out on the buildings and the estimated life and remaining life of the buildings were recorded. The component age is not an input parameter in this study for the focus of gamma process. However, to highlight the motivation of this study, these financial estimates are used to derive the age of the superstructure components. The scatter of deterioration vs. age is shown in Figure 01.

It is noted that the deterministic approach either linear or non-linear cannot be used to predict the deterioration of the superstructure with a sufficient accuracy. In addition, there are assumptions about the age of the elements that affect the accuracy of the prediction. 


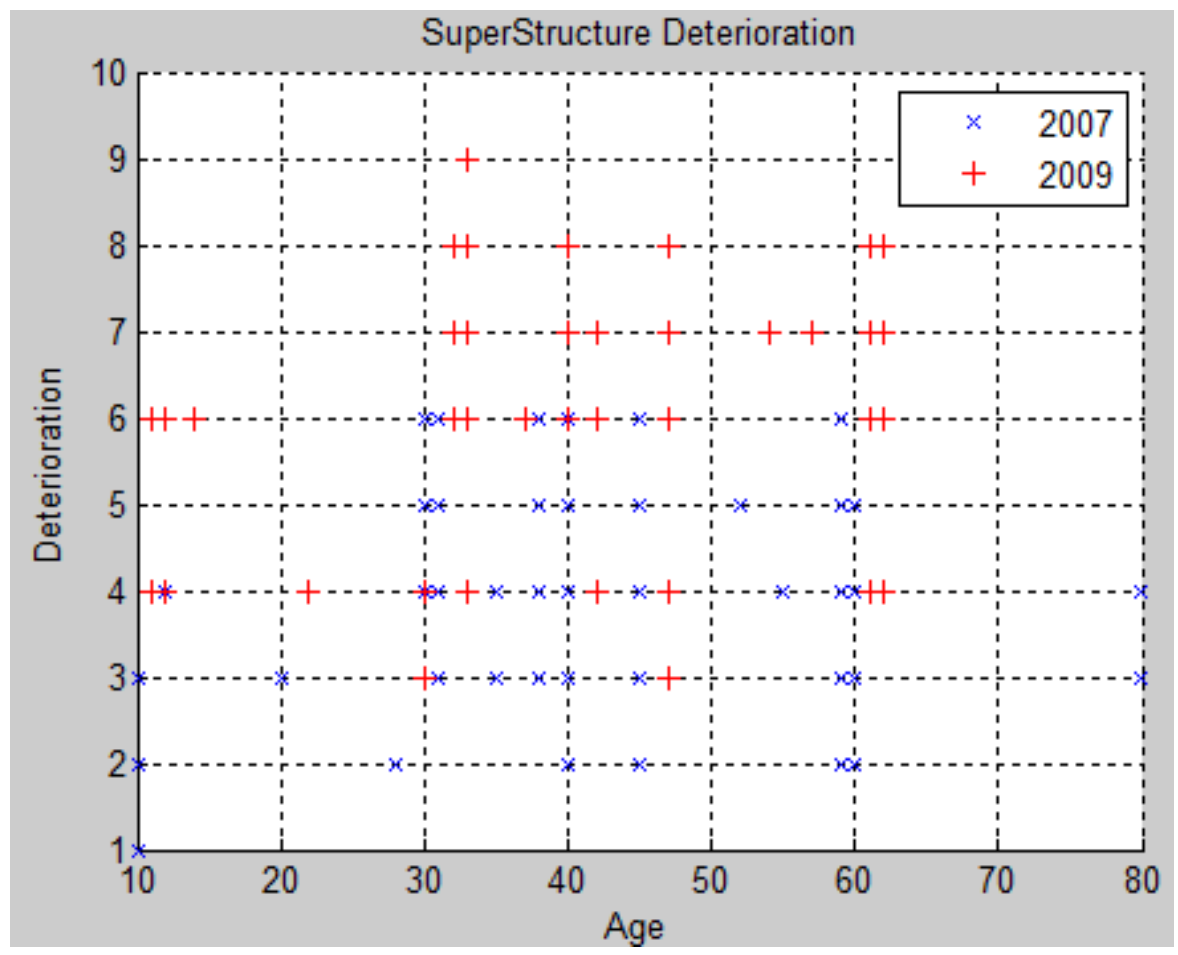

Figure 1: Superstructure Deterioration with age

\subsection{Parameter Estimation of the Gamma Process}

Modelling the temporal variability of the deterioration with a gamma process, the mean deterioration at time $t$ is often proportional to a power law as shown below.

$$
\mu_{X(t)}=\frac{a(t)}{b}=\frac{\alpha t^{\beta}}{b}
$$

Where $\mu_{X(t)}=\alpha>0, \beta>0$. The gamma process is stationary if the mean deterioration is linear in time, i.e. when $\beta=1$. The gamma process is non-stationary when $\beta \neq 1$.

\subsubsection{Method of Maximum Likelihood}

We used method of maximum likelihood to estimate the parameters $\alpha$ and $b$ of the stationary gamma process (where $\beta=1$ ). That is the logarithm of the likelihood function of the independent deterioration increments are maximized to obtain $\alpha$ and 
$b$. The likelihood function $L(\alpha, b)=\prod f_{i}\left(\delta_{i}\right)$ in which $f_{i}$ is the distribution of $\delta_{i}$ where $\delta_{i}=x_{i}-x_{i-1}$ in time interval $\theta_{i}=t_{i}-t_{i-1}$. Parameters are obtained by computing the first partial derivatives of the log likelihood function of the increments with respect to $\alpha$ and $b$, and solving the following equations:

$$
\frac{\partial}{\partial \alpha} L(\alpha, b)=0 ; \quad \frac{\partial}{\partial b} L(\alpha, b)=0 ;
$$

$L(\alpha, b)=\prod_{i} b^{\alpha\left(\theta_{i}\right)} \cdot \delta_{i}^{\alpha\left(\theta_{i}\right)-1} \cdot e^{-b \delta_{i}} / \Gamma\left(\alpha \theta_{i}\right)$ maximizing log $L$, and using Equation (9), following equations are obtained [25] for optimal values of $\hat{\alpha}$ and $\hat{b}$ (maximum likelihood estimates) for $\alpha$ and $b$.

$\hat{\alpha} \sum \theta_{i}=\hat{b} \sum \delta_{i}$ and

$$
\log \hat{b} \cdot \sum \theta_{i}+\sum \theta_{i} \log \delta_{i}=\sum \theta_{i} \Gamma^{\prime}\left(\hat{\alpha} \theta_{i}\right) / \Gamma\left(\hat{\alpha} \theta_{i}\right)
$$

Where $\Gamma^{\prime}(z)=d(\Gamma(x)) / d x$

Because the last inspection contains the most information, the expected deterioration at time $t$ can be derived based on the last inspections as below [17].

$$
E(X(t))=x_{n}\left(t / t_{n}\right)^{b}
$$

\subsection{Analysis of Data and Results}

$\alpha$ and $b$ are derived using the parameter estimation discussed above. $\alpha$ value is 0.1551 and $b$ is 0.1391 . The PDFs at various points in time t, are derived and shown in Figure 2. Corresponding CDFs are shown in Figure 3.

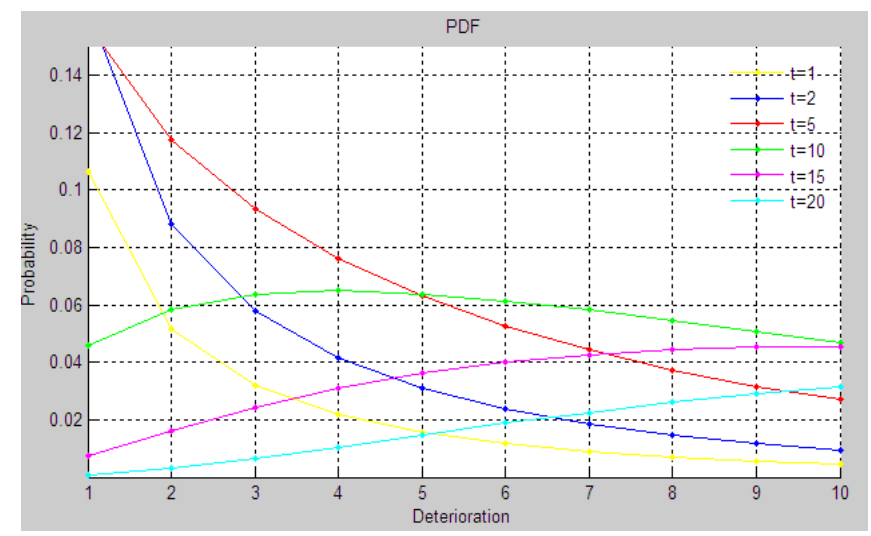

Figure 2: Gamma PDF of deterioration $\mathrm{X}(\mathrm{t})$ 
The Figure 4 illustrates the predicted deterioration over time. Deterioration at Year 1 corresponds to the mean value of the accumulated deterioration in 2009. Mean accumulated deterioration predicted for years from 2010 onwards correspond to the values of years from year 2 onwards. This prediction can be used for financial forecasting [2]. In addition, various procurement and management decision making can be done using this deterioration/condition based prediction as a base and incorporating other influencing factors such as social, economic, environmental and functional which is out of scope of this paper.

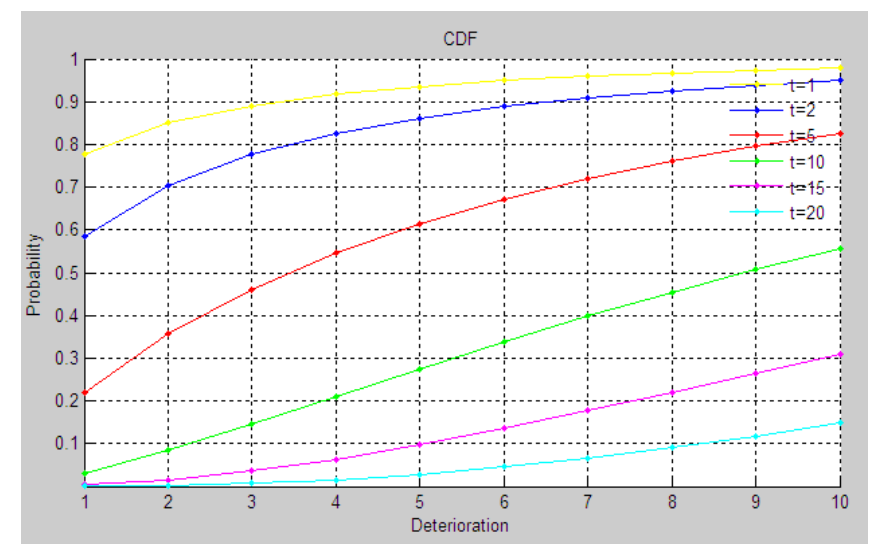

Figure 3: Gamma CDF of deterioration X(t)

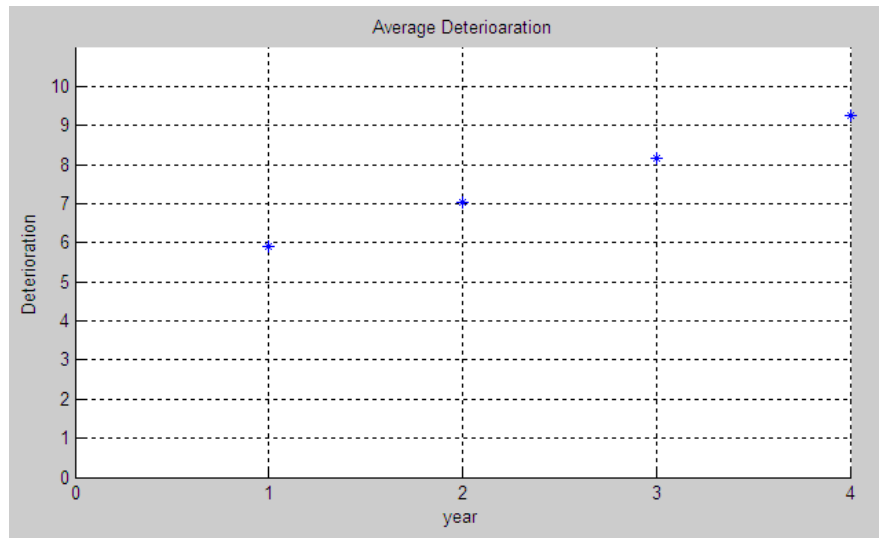

Figure 4: Predicted deterioration over time 


\section{Conclusions}

The paper has demonstrated the difficulties associated with fitting a deterministic deterioration prediction models to real condition data collected from council buildings. Reliability based methods of deterioration prediction have been reviewed and gamma process has been selected as a possible model to be examined. Application of the gamma process for forecasting condition deterioration of council buildings has been demonstrated with a real set of condition data collected form council buildings and using data from superstructure of buildings as an example. The ability of the Gamma process to forecast deterioration from condition data of high scatter has been demonstrated with the work reported here.

\section{Acknowledgement}

The authors gratefully acknowledge the insightful discussions and guidance provided by Dr. Malihe Abdollahiani at school of Math \& Geospatial Sciences at RMIT University.

\section{References:}

1. ABS. The Australian Bureau of Statistics estimates. 2001 [cited 2010 March ].

2. Edirisinghe, R., et al. Council Building Management practices, Case studies and Road Ahead. in The 5th World Congress on Engineering Asset Management (WCEAM). 2010. Brisbane, Australia.

3. Kleiner, Y. and B. Rajani, Comprehensive Review of Structural Deterioration of Water Mains: Statistical Models. Urban Water Journal, 2001. 3: p. 131-150.

4. Lou, Z., et al., Application of Neural Network Model to Forecast Short-Term Pavement Crack Condition: Florida Case Study. Journal of Infrastructure Systems, ASCE, 2001. 7(4): p. 166-171.

5. Wirahadikusumah, R., M.D. Abraham, and T. Iseley, Challenging Issues in Modeling Deterioration of Combined Sewers. Journal of Infrastructure Systems, ASCE, 2001. 7(2): p. 77-84.

6. Morcous, G., H. Rivard, and A.M. Hanna, Modeling Bridge Deterioration Using Case-Based Reasoning. Journal of Infrastructure Systems, ASCE, 2002. 8(3): p. 86-95.

7. Mishalani, R.G. and S.M. Madanat, Computation of Infrastructure Transition Probabilities Using Stochastic Duration Models. Journal of Infrastructure Systems, ASCE, 2002. 8(4): p. 139-148.

8. Hahn, M., R.N. Palmer, and M.S. Merrill. Prioritizing Sewer Line Inspection with an Expert System. in the 26th Annual Water Resources Planning and 
Management Conference WRPMD'99: Preparing for the 21st Century 1999. Tempe, Arizona.

9. Makropoulos, C.K., D. Butler, and C. Maksimovic, Fuzzy Logic Spatial Decision Support System for Urban Water Management. Journal of Water Resources Planning and Management, 2003. 129(1): p. 69-77.

10. Luis, F.S.F. and H. Naim, Neural Networks in Water Resources Management, in World Water Congress 2001, ASCE. 2001. p. 92-107.

11. Al-Barqawi, H. and T. Zayed, Condition Rating Model for Underground Infrastructure Sustainable Water Mains. Journal of Performance of Constructed Facilities, 2006. 20(2): p. 126-135.

12. Cattan, J. and J. Mohammadi, Analysis of Bridge Condition Rating Data Using Neural Networks. Computer-Aided Civil Engineering, ASCE, 1997. 12(6): p. 419-429.

13. Sinha, S.K. and M.D.Pandey, Probabilistic Neural Network for Reliability Assessment of Oil and Gas Pipelines. Computer-Aided Civil and Infrastructure Engineering, 2002. 17(5): p. 320-329.

14. Kim, D.K., et al., Application of Probabilistic Neural Networks for Prediction of Concrete Strength. Journal of Materials in Civil Engineering, ASCE, 2005. 17(3): p. 353-362.

15. Tran, H.D., Investigation of Deterioration Models for Storm Water Pipe Systems. 2007, Victoria University.

16. Madanat, S., R. Mishanlani, and W.H.W. Ibrahim, Estimation of infrastructure transition probabilities from condition rating data. Journal of Infrastructure Systems 1995. 19(2): p. 120-125.

17. vanNoortwijk, J.M., A survey of the application of gamma processes in maintenance. Reliability Engineering \& System Safety, 2009. 94: p. 2-21.

18. Pandey, M.D. and J.M. vanNoortwijk. Gamma process model for time-dependent structural reliability analysis. in Second International Conference on bridge maintanace, safety and management (IABMAS). 2004.

19. Madanat, S. and W.H.W. Ibrahim, Poisson Regression Models of Infrastructure Transition Probabilities. Journal of Transportation Engineering, ASCE, 1995. 121(3): p. 267-272.

20. Baik, H.S., H.S. Jeong, and D.M. Abraham, Estimating Transition Probabilities in Markov Chain-Based Deterioration Models for Management of Wastewater Systems. Journal of Water Resources Planning and Management, ASCE, 2006. 132(1): p. 15-24. 
21. Micevski, T., G. Kuczera, and P. Coombes, Markov Model for Storm Water Pipe Deterioration. Journal of Infrastructure Systems, ASCE, 2002. 8(2): p. 49-56.

22. Sharabah, A., S. Setunge, and P. Zeephongsekul, A Reliability Based Approach for Service Life Modeling of Council Owned Infrastructure Assets., in the ICOMS Asset Management Conference. 2007: Melbourne.

23. Singpurwalla, N.D. and S.P. Wilson, Failure models indexed by two scales. Advances in Applied Probability 1998. 30(4): p. 1058-72.

24. Abdel-Hameed, M., A gamma wear process. IEEE Transactions on Reliability, 1975. 24(2): p. 152-153.

25. Cinlar, E., Z.P. Bazant, and E. Osman, Stochastic process for extrapolating concrete creep. Journal of the Engineering Mechanics Division, 1977. 103: p. 1069-88.

26. Lawless, J. and M.Crowder, Covariates and random effects in a gamma process model with application to degradation and failure. Lifetime Data Analysis 2004. 10(3): p. 213-27.

27. Kallen, M.J. and J.M.v. Noortwijk, Optimal maintenance decisions under imperfect inspection Reliability Engineering and System Safety, 2005. 90(2-3): p. 177-85.

28. Frangopol, D.M., M.J. Kallen, and J.M.v. Noortwijk, Probabilistic models for life-cycle performance of deteriorating structures review and future directions. Progress in Structural Engineering and Materials, 2004. 6(4): p. 197-212.

29. Samali, B., K.I. Crews, and K.A. K., eds. A system for bridge network condition assessment and prediction Incorporating Sustainable Practice in Mechanics of Structure and Materials. 2011 Taylor \& Francis Group: London. 739-744.

30. Aboura, K., et al., Stochastic Processes for modeling bridge deterioration, in Futures in Mechanics of Structures and Materials. 2009 Taylor \& Francis Group: London. p. 533-538.

31. Frangopol, D.M., J.S. Kong, and E.S. Gharaibeh, Reliability-based life-cycle management of highway bridges. Journal of computing in civil Engineering, 2001. 15(1): p. 27-34. 
